Copyright by the American Physical Society. Marolf, D ; Minic, D ; Ross, SF, MAR 2004 . "Notes on spacetime thermodynamics and the observer dependence of entropy," PHYSICAL REVIEW D 69(6): 064006. DOI: 10.1103/PhysRevD.69.064006.

PHYSICAL REVIEW D 69, 064006 (2004)

\title{
Notes on spacetime thermodynamics and the observer dependence of entropy
}

\author{
Donald Marolf* \\ Physics Department, UCSB, Santa Barbara, California 93106, USA \\ Djordje Minic ${ }^{\dagger}$ \\ Institute for Particle Physics and Astrophysics, Department of Physics, Virginia Tech, Blacksburg, Virginia 24061, USA \\ Simon F. Ross ${ }^{\ddagger}$ \\ Centre for Particle Theory, Department of Mathematical Sciences, University of Durham, South Road, \\ Durham DH1 3LE, United Kingdom \\ (Received 11 November 2003; published 10 March 2004)
}

\begin{abstract}
Because of the Unruh effect, accelerated and inertial observers differ in their description of a given quantum state. The implications of this effect are explored for the entropy assigned by such observers to localized objects that may cross the associated Rindler horizon. It is shown that the assigned entropies differ radically in the limit where the number of internal states $n$ becomes large. In particular, the entropy assigned by the accelerated observer is a bounded function of $n$. General arguments are given along with explicit calculations for free fields. The implications for discussions of the generalized second law and proposed entropy bounds are also discussed.
\end{abstract}

DOI: 10.1103/PhysRevD.69.064006

PACS number(s): 04.70.Dy, 04.62.+v

\section{INTRODUCTION}

The interaction of thermodynamics with gravitation has been a subject of intense interest since the discovery of black hole entropy [1] and Hawking radiation [2]. The geometrical entropy associated with black hole horizons is thought to provide an important clue to the quantum structure of spacetime, and many attempts have been made to parse the corresponding riddle. The approach most reminiscent of ordinary statistical mechanics attempts to identify a collection of microstates whose counting reproduces this entropy. Despite some successes, particularly in the context of string theory [3], we are still far from having a concrete understanding of these states in the regime where the classical geometrical description of a black hole is a good approximation to the underlying physics (and we do not aim to improve this situation in the present paper).

Other authors have tried to extend black hole entropy to more general principles. In particular, the conjecture that the relation between entropy and area extends to any event horizon (for example, to acceleration horizons in flat space) has been recently championed by Jacobson [4] and by Jacobson and Parentani [5] who studied the associated first law of thermodynamics. ${ }^{1}$ An interesting output of this line of reasoning is the suggestion [4] that horizon entropy arises because gravity is thermodynamics (or a special case thereof). Adopting an alternative approach, a number of authors [7-10] have argued that the consistency of the second law of thermodynamics with a black hole entropy related to the area of the event horizon yields a general principle restricting the

\footnotetext{
*Email address: marolf@physics.syr.edu

${ }^{\dagger}$ Email address: dminic@vt.edu

\#Email address: S.F.Ross@durham.ac.uk

${ }^{1}$ Other work with this theme includes [6] and references therein.
}

allowed entropy of any system, be it black hole, matter, or other.

Our aim in this paper is to explore a new relation between horizons and entropy, which we believe is relevant to the discussion above. We will show that the entropy associated with a simple localized matter system in flat and otherwise empty space is not an invariant quantity defined by the system alone, but rather depends on which observer we ask to measure it. An inertial observer will assign the usual, naive entropy given by the logarithm of the number of internal states. However, an accelerated observer (who sees the object immersed in a bath of thermal radiation) will find the object to carry a different amount of entropy. Note that in the context we will consider both observers are able to describe the object with the same degree of precision; the issue is not that our object is partially hidden behind the Rindler horizon.

It is of course well known that the inertial and Rindler observers already ascribe a different entropy to the Minkowski vacuum, as this is a thermal state with divergent entropy [11] from the Rindler point of view. Considering both this fact and the background structures necessary for standard discussions of thermodynamics, Wald has argued for some time [12] (see also the last part of [13]) that entropy is an extremely subtle concept in general relativity-even for ordinary matter systems - and that we still lack the proper framework for a general discussion. Our results are in complete agreement with this philosophy and may be considered a next small step in pursuit of this goal. In particular, we now learn that the observer dependence of entropy is far more than a simple shift of the zero point. What is perhaps surprising from the point of view of [12] is that the observers can disagree on the entropy of a localized object even when they assign the same energy to all of its microstates.

The fact that an accelerated observer does not measure the usual naive entropy for an inertial matter system clearly has important consequences for thermodynamic discussions, and 
our motivation for investigating these questions is closely related to recent explorations of such issues. Let us therefore recall the recent progress $[14,15]$ in clarifying issues related to the 2 nd law as raised in [7-10]. To review the basic question, suppose one considers a black hole of temperature $T$ and mass $M$. Classically, a mass $M$ black hole will absorb any small object placed nearby. ${ }^{2}$ If an object $(o b j)$ carries energy $E_{o b j}$ into the black hole the associated increase in black hole entropy will be $d S=E_{o b j} / T$ by the first law. But what if the small object itself has more entropy $S_{o b j}$ than $E_{o b j} / T$ ? Then such an absorption would violate the generalized 2nd law.

But let us also recall that [16] argues that the generalized 2nd law will in general be satisfied when the system outside the black hole can be described by quantum field theory in curved spacetime. Though issues of divergences and back reaction prevent this from being a rigorous proof, it is at least highly suggestive. If correct, then there are two logical alternatives. Either objects with $S_{o b j}>E_{o b j} / T$ cannot exist in any consistent quantum field theory (in curved spacetime), or some quantum effect must intervene to increase the entropy of the final state.

The latter conclusion was recently argued in $[14,15]$. The main point was that for $S>E / T$ either (i) thermally produced objects macroscopically indistinguishable from that which one is attempting to insert into the given black hole will play an important role in the thermal atmosphere of this black hole, or (ii) the object of interest will be unable to freely penetrate this black hole's thermal atmosphere. There is a large overlap between the two cases; for example, the presence of similar objects in the thermal atmosphere can cause the new object one introduces to experience a repulsive pressure. Such an effect might come either through an explicit interaction or through statistical effects (e.g., the Pauli exclusion principle). This repulsive pressure can prohibit our object from falling into the black hole.

In the case where our object can freely penetrate the thermal atmosphere, [15] suggested how quantum effects could still alter the final state. The point is that, as mentioned above, the thermal atmosphere must contain a significant number of such objects. This is argued [15] by computing the free energy $F_{o b j}=E_{o b j}-T S_{o b j}$ of such an object at the Hawking temperature $T$ and finding that $F_{o b j}$ is negative. Since the vacuum has zero free energy (i.e., a greater amount), at temperature $T$ our objects are more likely to exist than not.

Suppose now that the black hole were to come to equilibrium (say, if placed in a small reflecting cavity) with its thermal atmosphere. Such an equilibrium state would contain a significant flux of objects directed toward the black hole. But since there is equilibrium, it must be that similar objects are also radiated from the black hole at a significant rate. Reference [15] argues that this will be the case even for large $E_{o b j} / T$ due to the fact that the entropy $S_{o b j}$ has been assumed even larger.

This effect appears to be sufficient to protect the second

\footnotetext{
${ }^{2}$ Though classically such a black hole will have $T=0$.
}

law when one drops a single $S_{o b j}>E_{o b j} / T$ object (or a small number of such objects) into a black hole in otherwise empty space. But there remains a final wrinkle to sort out: what if we measure the flux of such objects being radiated from the black hole and then arrange to beam our objects into the black hole at a higher rate? Or, suppose we place the black hole in a reflecting cavity, let it reach equilibrium, and then send in another object with more than $e^{E_{o b j} / T}$ internal states?

Since the density of such objects in equilibrium may already be high, this may require us to assume extremely weak interactions. But this is not a problem in principle, and in the present work we focus on the case of free fields. In the limit of a large black hole, the problem reduces to the study of a Rindler horizon in flat spacetime. The equilibrium state is just the Minkowski vacuum which, however, appears thermally excited to uniformly accelerated (i.e., Rindler) observers.

Here, the observer dependence of entropy becomes crucial. We find that inertial and Rindler observers do not ascribe the same amount of entropy to our object. Inertial observers ascribe an entropy equal to the logarithm of the number of internal states, as expected. However, in the limit where the number of internal states $n$ is large, a Rindler observer ascribes only an entropy $S_{a c c}=E_{a c c} / T,{ }^{3}$ suggesting consistency of the second law ${ }^{4}$ when such an object crosses the event horizon. Thus, a consequence of our observer dependence of entropy is that allowing an object to fall across an event horizon will plausibly respect the generalized second law from the point of view of the accelerated observer who remains outside of the horizon, no matter how many internal states the object carries. However, our analysis stops short of being a proof for reasons related to the unresolved issues with [16]; these are discussed further in the concluding section. (The inertial observer, on the other hand, never loses sight of the object, so there can be no question of a violation of the second law from their point of view.)

We will give a general argument for this observer dependence of the entropy in the next section. Our argument relies simply on general points about thermodynamics and the relation of entropy to statistical ensembles. We will see that the Rindler observer ascribes an entropy $S_{a c c}=E_{a c c} / T$ to the object whenever it represents a small perturbation on the thermal state, which in particular will be true in the limit of a large number of microstates. Section III then fleshes out the detailed calculations in the case of free boson and free fermion fields. In particular, the relation between the Rindler energy $E_{a c c}$ of the object and the inertially measured energy $E_{\text {inertial }}$ is calculated for a well-localized object. With the proper normalization of $E_{a c c}$, and in the limit $E_{\text {inertial }} \ll T$,

\footnotetext{
${ }^{3}$ Here $E_{a c c}$ (the energy measured by the accelerated observer) is the Killing energy associated with the boost symmetry $\xi$ (i.e., the Rindler time translation). The associated temperature $T$ is given by $T=\kappa / 2 \pi$ where $\kappa$ is the surface gravity of $\xi$. As usual, the normalization of $\xi$ cancels so that $E_{a c c} / T$ is independent of this choice.

${ }^{4}$ Either the second law for black holes in the limit of a large black hole, or the "stationary comparison" second law for asymptotic Rindler horizons [5].
} 
one finds $E_{a c c}=E_{\text {inertial }}$ as one would expect. However, for larger $T$ the answer can be rather different. We close with some discussion in Sec. IV.

\section{ENTROPY AND OBSERVERS}

The central point of this paper is to show that the entropy ascribed to a localized physical system by different observers can differ, even when both observers describe the region containing the system with the same resolution. We exhibit this in a particularly simple context, considering the entropy of an object in flat space as seen by an inertial or a Rindler observer. Note that we take the spacetime to be exactly flat and explicitly ignore any possible gravitational effects from the objects we discuss. We will return to this point in Sec. IV. In this section, we give a general argument suggesting that the entropy measured by the Rindler observer will be bounded by $E_{a c c} / T$ and strongly arguing for this result in the limit of a large number of internal states. Here $E_{a c c}$ is the energy associated to the object by the Rindler observer and $T$ temperature seen by this observer. In the following section, we give some more detailed calculations for the case of single-particle excitations of free Bose or Fermi fields.

From the inertial observer's point of view, the natural vacuum state is the Minkowski vacuum $\left|0_{M}\right\rangle$, and we describe the excitation of this vacuum corresponding to the presence of some object (in an undetermined microstate) by the density matrix

$$
\rho_{M}=\frac{1}{n} \sum_{i=1}^{n}\left|1_{M} ; i\right\rangle\left\langle 1_{M} ; i\right|
$$

where the $\left|1_{M} ; i\right\rangle$ denote Minkowski one-object states describing the $n$ possible microstates of our object. The entropy assigned to the object by the inertial observer is then as usual

$$
S_{M}=-\operatorname{Tr} \rho_{M} \ln \rho_{M}=\ln n .
$$

But what entropy will a Rindler observer assign to this object? Note that the answer is not given by $-\operatorname{Tr} \rho \ln \rho$ where $\rho$ is the density matrix corresponding to $\rho_{M}$ from the Rindler observer's point of view. This $-\operatorname{Tr} \rho \ln \rho$ would include in addition a contribution from the background of thermal acceleration radiation that the Rindler observer may not wish to ascribe to the localized object. One way to define the entropy of the object from the Rindler observer's point of view is to ask what entropy is carried out of the visible Rindler wedge when the object crosses the Rindler horizon. ${ }^{5}$ Thus, the appropriate Rindler notion of entropy $S_{\text {acc }}$ carried by the object is the difference $\delta S$ between the entropy in the visible wedge when the object of interest is present and when it is not. Similarly $E_{a c c}=\delta E$ is the corresponding difference in Killing energies. We repeat that we work in the approxi-

\footnotetext{
5The terms "visible" and "invisible" Rindler wedge will always be used in the context of what is in causal contact with our chosen Rindler observer. Of course, the entire spacetime is visible to the inertial observer.
}

mation where gravitational back reaction is neglected and, in particular, in which the horizon is unchanged by the passage of our object.

We therefore first consider the thermal density matrix $\rho_{R 0}$ which results from tracing the Minkowski vacuum, $\left|0_{M}\right\rangle\left\langle 0_{M}\right|$, over the invisible Rindler wedge:

$$
\rho_{R 0}=\operatorname{Tr}_{\text {invisible }}\left|0_{M}\right\rangle\left\langle 0_{M}\right| \text {. }
$$

This describes all information that the Rindler observer can access in the Minkowski vacuum state. We wish to compare $\rho_{R 0}$ with another density matrix $\rho_{R 1}$ which provides the Rindler description of the state $\rho_{M}$ above, in which one object (in an undetermined microstate) is added to the Minkowski vacuum $\left|0_{M}\right\rangle$. The density matrix $\rho_{R 1}$ is hence

$$
\rho_{R 1}=\operatorname{Tr}_{\text {invisible }} \rho_{M}=\operatorname{Tr}_{\text {invisible }} \frac{1}{n} \sum_{i=1}^{n}\left|1_{M} ; i\right\rangle\left\langle 1_{M} ; i\right| .
$$

We would like to compute the difference in energy

$$
\delta E=\operatorname{Tr}\left[H\left(\rho_{R 1}-\rho_{R 0}\right)\right],
$$

and entropy

$$
\delta S=-\operatorname{Tr}\left[\rho_{R 1} \ln \rho_{R 1}-\rho_{R 0} \ln \rho_{R 0}\right],
$$

where $H$ is the Hamiltonian of the system and, in both cases, the sign has been chosen so that the change is positive when $\rho_{R 1}$ has the greater value of energy or entropy. The entropy of each state separately is well known to be divergent [11]. However, there is no reason to expect $\delta S$ to be ill-defined in our context. Assuming that the object has some moderately well-defined inertially-measured energy $E_{\text {inertial }}$ and is well localized, the energy measured by the Rindler observer will also be reasonably well-defined and the difference $\rho_{R 1} \ln \rho_{R 1}-\rho_{R 0} \ln \rho_{R 0}$ will have negligibly small diagonal entries at high energy so that the above trace will exist. In other words, we may compute $\delta S$ by first imposing a cutoff $\Lambda$, computing the entropy $\left(S_{0}, S_{1}\right)$ and energy $\left(E_{0}, E_{1}\right)$ of the two states $\left(\rho_{R 0}, \rho_{R 1}\right)$ separately, subtracting the results, and removing the cutoff. We will see that the cutoff dependence is trivial in the approximation used below.

Let us make the simplifying assumption that the object represents a small perturbation on the initial thermal Rindler density matrix $\rho_{R 0}$; that is, $\rho_{R 1}=\rho_{R 0}+\delta \rho$, where " $\delta \rho$ $\ll \rho_{R 0}$." One expects this approximation to hold for $n e^{-E / T}$ $\gg 1$, in which case (on average) there are already many objects similar to ours present in $\rho_{R 0}$. In particular, this approximation should hold in the limit of large $n$ with fixed $E / T$. A somewhat more careful argument for this approximation was explained to us by Mark Srednicki and is given in the Appendix.

We therefore approximate $\delta S$ by Taylor expanding around $\rho_{R 0}$ 


$$
\begin{aligned}
\delta S & \approx-\operatorname{Tr}\left[\left.\delta \rho \frac{\delta(\rho \ln \rho)}{\delta \rho}\right|_{\rho=\rho_{R 0}}\right] \\
& =-\operatorname{Tr}\left[\delta \rho\left(1+\ln \rho_{R 0}\right)\right]=-\operatorname{Tr}\left[\delta \rho \ln \rho_{R 0}\right],
\end{aligned}
$$

where in the last step we use the fact that $\operatorname{Tr} \rho_{R 1}=\operatorname{Tr} \rho_{R 0}$. Now recall that the initial density matrix is thermal, $\rho_{R 0}$ $=e^{-H / T} /\left(\operatorname{Tr} e^{-H / T}\right) ;$ as a result

$$
\begin{aligned}
\delta S & \approx-\operatorname{Tr}[\delta \rho(-H / T)] \\
& =\frac{\operatorname{Tr}\left[\left(\rho_{R 1}-\rho_{R 0}\right) H\right]}{T}=\frac{\delta E}{T},
\end{aligned}
$$

where we have again used $\operatorname{Tr}(\delta \rho)=0$. This key result is independent of any cutoff.

This result can also be understood on the basis of classical thermodynamic reasoning. The initial configuration $\rho_{R 0}$ represents a thermal equilibrium. We wish to calculate the change in entropy in a process which increases the energy by an amount $\delta E$. Whatever the nature of the object that we add, this cannot increase the entropy by more than it would have increased had we added this energy as heat. Since we consider a small change in the configuration, the first law yields

$$
\delta S<\delta S_{\max }=\frac{\delta E}{T}
$$

We see that the process of adding a small object saturates this bound, at least to first order in small quantities. We should expect that if we relaxed the assumption that the object represents a small change in the configuration, the resulting $\delta S$ will satisfy but no longer saturate the integrated version of this bound.

We have found that the entropies ascribed to the localized object by Minkowski and Rindler observers behave in quite different ways. From the point of view of the Minkowski observer, entropy and energy are independent; the object can have an arbitrarily large entropy with fixed energy if it has sufficiently many internal states. On the other hand, from the point of view of the Rindler observer, entropy and energy are linked in a very general way by the bound (2.9). As we will see in the examples in the next section, the Rindler entropy for "highly entropic objects" will be much smaller than the inertial entropy.

Note that since it arises from a trace over the invisible Rindler wedge, this restriction is generally correlated with the presence of a horizon, to which we might want to assign a geometric entropy. Thus, this reduction in the ascribed entropy is important for a complete understanding of attempts to violate the generalized second law using such highly entropic objects, as reviewed in the Introduction. We will return to such issues in Sec. IV.

\section{CALCULATIONS FOR FREE FIELDS}

We now explore two concrete examples to illustrate and elucidate the general discussion above. We consider a system of $n$ free Bose or Fermi fields, and calculate the entropy of objects described, from the inertial point of view, by a singleparticle density matrix uniformly distributed over the different fields. Thus the inertial observer assigns the object an entropy $S_{\text {inertial }}=\ln n$ in each case.

\section{A. Bosons}

For a system of $n$ free bosonic fields, the Minkowski vacuum can be recast in terms of the Rindler data as the following entangled state [17]:

$$
\left|0_{M}\right\rangle=\prod_{i=1}^{n} \prod_{j}\left(1-e^{-\omega_{j} / T}\right)^{1 / 2} e^{-\left(\omega_{j} / 2 T\right) a_{i j L}^{\dagger} a_{i j R}^{\dagger}\left|0_{\text {Rindler }}\right\rangle .}
$$

Here $i$ labels the different fields, $j$ labels a complete set of modes $u_{j L}, u_{j R}$ of positive Rindler frequency $\omega_{j}$ for each field, and the labels $R$ and $L$ refer respectively to the right and left Rindler wedges. Each mode $u_{i L}, u_{j R}$ of the $i$ th field has an associated annihilation operator $a_{i L}, a_{i R}$ which annihilates the Rindler vacuum $\left|0_{\text {Rindler }}\right\rangle$ and satisfies a standard commutation relation of the form $a a^{\dagger}-a^{\dagger} a=1$. The temperature $T$ associated with the uniformly accelerating observer of interest is given by $T=a / 2 \pi=\kappa / 2 \pi$ where $a$ is the observer's proper acceleration and $\kappa$ is the surface gravity of the boost Killing field $\xi$ that is normalized on our observer's world line.

Similarly, the annihilation operator $a_{i M}$ for a Minkowski mode $u_{M}$ of the $i$ th field can on general principles [17] be expressed in the form

$$
\begin{aligned}
a_{i M}= & \sum_{j}\left[\left(u_{M}, u_{j R}\right)\left(a_{i j R}-e^{-\omega_{j} / 2 T} a_{i j L}^{\dagger}\right)\right. \\
& \left.+\left(u_{M}, u_{j L}\right)\left(a_{i j L}-e^{-\omega_{j} / 2 T} a_{i j R}^{\dagger}\right)\right],
\end{aligned}
$$

where $(u, v)$ is the Klein-Gordon inner product. For simplicity we might suppose that we choose a mode $u_{M}$ with no support on the invisible Rindler wedge (say, the left one) and for which $\left(u_{M}, u_{j R}\right)$ is well modeled by a delta function; we will return to the general case later in the subsection. In particular, this simplification means that the Rindler frequency $\omega$ of $u_{M}$ is reasonably well defined. ${ }^{6}$ For such a case the above Bogoliubov transformation becomes

$$
a_{i M}=\frac{1}{\sqrt{1-e^{-\omega / T}}}\left(a_{i R}-e^{-\omega / 2 T} a_{i L}^{\dagger}\right)
$$

where the normalization of $a_{i M}$ is fixed by the commutation relation. Here the Rindler operators refer to the one relevant pair of Rindler modes.

Since $a_{i M}$ acts in the Hilbert space describing only the $i$ th field, it is convenient to describe the action of $a_{i M}$ on the vacuum $\left|0_{i M}\right\rangle$ for this particular field alone. One sees that the

\footnotetext{
${ }^{6}$ If the object is well localized and located near our Rindler observer at some time, then $\omega$ is also the frequency measured by the inertial observer.
} 
properly normalized Minkowski one-particle state is given in terms of an infinite number of Rindler excitations

$$
a_{i M}^{\dagger}\left|0_{i M}\right\rangle=\left(1-e^{-\omega / T}\right) \sum_{k} e^{-k \omega / 2 T} \sqrt{k+1}|k, k+1\rangle_{i},
$$

where $|k, k+1\rangle_{i}$ denotes the state of field $i$ having $k$ Rindler excitations in the right wedge and $k+1$ Rindler excitations in the left wedge. Here we consider only the factor in the Hilbert space that describes the modes appearing in Eq. (3.3).

Thus, if we return to our Minkowski density matrix

$$
\begin{aligned}
\rho_{M} & =\frac{1}{n} \sum_{i=1}^{n}\left|1_{M} ; i\right\rangle\left\langle 1_{M} ; i\right| \\
& =\frac{1}{n} \sum_{i=1}^{n}\left(a_{i M}^{\dagger}\left|0_{M}\right\rangle\left\langle 0_{M}\right| a_{i M}\right),
\end{aligned}
$$

tracing over the invisible Rindler wedge will give the desired result. A short calculation gives

$$
\rho_{R 1}^{\text {diag }}=\operatorname{Tr}_{\text {invisible }} \rho_{M}=\left(e^{\omega / T}-1\right) \frac{1}{n} \sum_{i} N_{i} \rho_{R 0},
$$

where $\rho_{R 0}$ is the original density matrix corresponding to the Minkowski vacuum, $\rho_{R 0}=\operatorname{Tr}_{\text {invisible }}\left|0_{M}\right\rangle\left\langle 0_{M}\right|$, and $N_{i}$ denotes the number operator for the $i$ th field. The superscript diag indicates that we have written only the diagonal part of $\rho_{R 1}$ in the standard basis, which the reader will shortly see is all that contributes to $\delta E$ and $\delta S$ below. One can check explicitly that $\rho_{R 1}$ is properly normalized so that its trace is 1.

The change in the density matrix is thus $\delta \rho=\rho_{R 1}-\rho_{R 0}$. As in the previous section, we assume that this change is small, so that we can compute the change in entropy by $\delta S$ $=-\operatorname{Tr}(\delta \rho(1+\ln \rho))$. For noninteracting particles, the Hamiltonian is $H=\Sigma_{i} N_{i} \omega$, so the average change in energy is

$$
\delta E=\omega \operatorname{Tr}\left(\sum_{i} N_{i} \delta \rho\right)=\frac{\omega}{1-e^{-\omega / T}} .
$$

This result is already of interest. Note that for $\omega \gg T$ and under the conditions of footnote 6 , one finds $\delta E=E_{\text {inertial }}$ $\approx \omega$. On the other hand, for $\omega \ll T$ the background of objects in the thermal bath leads to an amplification reminiscent of the effect of stimulated emission. ${ }^{7}$

On the other hand, the change in entropy is

$$
\begin{aligned}
\delta S & =-\operatorname{Tr}\left[\delta \rho\left(1+\ln \rho_{R 0}\right)\right] \\
& =-\operatorname{Tr}\left[\delta \rho\left(1+C-\frac{\omega N}{T}\right)\right]=\frac{\delta E}{T},
\end{aligned}
$$

\footnotetext{
${ }^{7}$ Interesting comments on the emergence of the Rindler thermal fluctuations can be found in [18].
}

where in the second step we used that $\rho_{R 0}$ is thermal (i.e., $\rho_{R 0}=C e^{-H / T}$, where $C$ is a c-number); and in the final step we use $\operatorname{Tr} \delta \rho=0$. It is clear that Eqs. (3.7) and (3.8) depend only on the diagonal part of $\rho_{R 1}$ since the other factors in the trace are all diagonal.

This expression obviously saturates the bound provided by the second law of thermodynamics in agreement with the general arguments of Sec. II. The results are independent of the number of species $n$ due to the fact that both $\delta E$ and $\delta S$ are linear in $\delta \rho$, while $\delta \rho$ is a normalized average over terms in which each field is changed independently. Thus for large $n$ the Rindler observer will ascribe a much smaller entropy to the object.

Finally, let us return to the case where more than one pair of Rindler modes contributes to Eq. (3.2). Since $\rho_{M}$ is quadratic in $a_{i M}$, this leads to two sums over Rindler operators in Eq. (3.5). However, since $N_{i}$ and $\left(1+\ln \rho_{R 0}\right)$ are diagonal in the standard Rindler Fock basis, inspection of Eqs. (3.7) and (3.8) shows that only the corresponding diagonal part of $\rho_{M}$ will contribute to $\delta E$ and $\delta S$. This diagonal part contains only a single sum over modes, weighted by $\left|\left(u_{M}, u_{j R}\right)\right|^{2}$ or $\left|\left(u_{M}, u_{j L}\right)\right|^{2}$. In the case where the Minkowski mode has no support in the left wedge (so that $\left|\left(u_{M}, u_{j L}\right)\right|^{2}=0$ ), the effect is just as if each mode represented a separate field. But we have seen that the final result is independent of the number of fields and, by the same logic, it is independent of the weighting given to the various fields in Eq. (3.5), so long as $\rho_{M}$ is properly normalized. Thus we again obtain Eqs. (3.7) and (3.8). In fact, it is clear that spreading the support over many modes only helps to justify our approximation as it increases the effective number of fields. In the more general case where the Minkowski mode function overlaps the invisible wedge, the quantities $\delta E$ and $\delta S$ will be reduced in proportion to the probability that the object lies in the invisible wedge but one again finds $\delta S=\delta E / T$.

\section{B. Fermions}

For noninteracting fermions the calculation proceeds in a very similar fashion. The Minkowski vacuum written as an entangled state is now

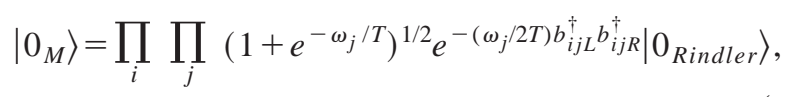

where the only changes from before are that the $b$ operators are fermionic with $b b^{\dagger}+b^{\dagger} b=1$, and the associated change in sign in the thermal normalization factor.

As for the boson field, working to first order in $\delta \rho$ makes $\delta E$ and $\delta S$ independent of the number of species $n$. As a result, we may take $n=1$ to evaluate the linearized result. Though the linearized approximation is in general valid only for large $n$, this does not prevent us from calculating the linearized result for $n=1$ and using it to correctly compute the large $n$ result. Similarly, since only the diagonal part of $\delta \rho$ will contribute we will also obtain the correct result (for an object whose wave function vanishes in the invisible wedge) by supposing that our Minkowski mode overlaps 
only a single pair of Rindler modes. The associated Bogoliubov transformation then takes the form

$$
b_{M}=\frac{1}{\sqrt{1+e^{-\omega / T}}}\left(b_{L}+e^{-\omega / 2 T} b_{R}^{\dagger}\right) .
$$

For a single field, the Rindler description of the Minkowskian one particle state is simply

$$
b_{M}^{\dagger}\left|0_{M}\right\rangle=|1,0\rangle_{\text {Rindler }},
$$

with one particle in the visible wedge and none in the invisible wedge. The properly normalized density matrix is just $\rho_{R 1}=|1\rangle\langle 1|$. Hence, the change in the Rindler density ma$\operatorname{trix} \delta \rho$ is

$$
\delta \rho=-\left(1+e^{-\omega / T}\right)^{-1}(|0\rangle\langle 0|-| 1\rangle\langle 1|),
$$

and the change in energy for a noninteracting system of excitations is

$$
\operatorname{Tr}(H \delta \rho)=\frac{\omega}{1+e^{-\omega / T}}
$$

In general, one now finds $\delta E<E_{\text {inertial }}$ so that $\delta S$ $\ll S_{\text {inertial }}$ for large $n$. When the assumptions of footnote 6 hold and $\omega \gg T$, one also finds $\delta E=E_{\text {inertial }} \approx \omega$, but for $\omega$ $\ll T$, the background thermal bath of fermions leads to a suppression of the Rindler energy. Said differently, the appearance of an object from the inertial point of view can sometimes correspond to the disappearance of an object from the Rindler observer's thermal bath.

Since $\delta \rho$ is traceless, one again finds $\delta S=\delta E / T$, saturating the bound provided by the first and second laws.

\section{DISCUSSION}

We have argued that inertial and accelerated observers naturally ascribe different values to the entropy of a localized matter system in flat Minkowski space, even if the system is fully visible to both observers and the observers describe the system with the same resolution. Certainly, the observers measure a different $\delta S$ when the localized system is introduced or removed, and so must use different values when considering a thermodynamic accounting of such processes. The results support the suggestions of [12] that entropy, even of ordinary matter systems, is a very subtle concept in general relativity or quantum field theory in curved spacetime.

What is surprising from the point of view of [12] is that our effect occurs even when the observers agree on the energy of each microstate. Our effect is in some sense due to a "mixing" between the object considered and the thermal background seen by the Rindler observer. In particular, we argued that when the number of microstates $n$ satisfies $n e^{-E / T} \gg 1$ the entropy assigned to our object by the accelerated observer is $\delta S=\delta E / T$. Note that in this regime a thermal ensemble of distinguishable particles would diverge, as each new particle would add an entropy $\ln n \gg E / T$. Thus, statistics plays an important role and one cannot rely on any intuition built from the study of distinguishable particles.

The above sections provided general arguments as well as explicit calculations for free fields. It is clear that the general arguments apply equally well to static observers in the presence of a black hole or a cosmological (e.g., de Sitter) horizon, and that the free field calculations would take a form that is essentially identical. Our general arguments are quite similar to those of [16], and our observer dependence of entropy provides an important physical mechanism behind such arguments. We note that the theorem derived in [16] applies rigorously in the context considered here in which the temperature and the location of the horizon are held constant and the dependence on any cutoff is trivial.

In the black hole case, the result $\delta S=\delta E / T$ would apply to any static observer and would in general differ from the entropy assigned by a freely falling observer. It is therefore interesting to return to the discussion of attempts to violate the generalized second law (i.e., including horizon entropy) by dropping an object with $n \gg e^{E / T}$ microstates into a black hole. Suppose first that the object begins far away and that we work in asymptotically flat space. Then the object will begin in a region of space that is not in thermal equilibrium with the black hole and where the effective local temperature vanishes. Here a static observer measures the object to have entropy $\ln n$ since there is no thermal bath. On the other hand, as the object approaches the black hole horizon one expects that it enters a region which is (nearly) at thermal equilibrium at the black hole temperature $T$ as described by the distant static observer. If the object acts like a free field, ${ }^{8}$ we have seen that at this point the static observer can ascribe only an entropy $S=E / T$ to the object. Thus if nothing else occurs the second law will indeed have been violated. The important point, however, is that this will occur before the object reaches the horizon itself. Thus, one expects that the generalized second law is protected by the same mechanism that protects the ordinary second law. In the present case, one expects to see a large flux of objects radiated by the black hole as, inverting the above argument, movement of highly entropic objects from the warm near-horizon neighborhood into the cold region of space will dramatically increase the entropy.

The effect is similar to attempting to send a low frequency photon (with two internal states) into a hot cavity which is otherwise at equilibrium-many more photons will emerge. Sending in a larger flux of photons does not help as a beam with $N$ photons does not in general have entropy $N \ln 2$ unless the photons are well separated, but well-separated photons will never overpower the large outward flux. This latter effect is also important for AdS black holes which can be at equilibrium with the entire spacetime.

As with [16], this result suggests that the generalized second law holds whenever the region outside the black hole is described by standard quantum field theory, regardless of whether one imposes bounds of the forms suggested in [7-10]. However, both stop short of being proofs because

\footnotetext{
${ }^{8}$ And thus falls freely so that the energy is constant in the test object approximation.
} 
they do not trace the actual dynamical changes as an object falls through the horizon. The absorption of the object by the black hole will change both the temperature and the size of the horizon. Since a change of the horizon size in some sense implies a change in the "location" of the horizon, any proof built without a detailed theory of quantum gravity would likely require careful assumptions as to the treatment of modes near the horizon which, by themselves, lead to a divergence in the total entropy $-\operatorname{Tr} \rho \ln \rho$ of the thermal bath seen by the accelerated observer [11]. For a finite (as opposed to infinitesimal) process, careful consideration would need to be given to nonequilibrium issues. Some analysis of the effect on the horizon was reported in [5], but further progress in this direction would be of use.

\section{ACKNOWLEDGMENTS}

The stimulating atmosphere of the Aspen Center of Physics, where this work was initiated, is gratefully acknowledged. D.M. would also like to thank Ted Jacobson and Mark Srednicki for interesting discussions. D.M. was supported in part by NSF grant PHY03-54978, and by funds from the University of California. S.F.R. was supported by the EPSRC.

\section{APPENDIX: EVALUATION OF $\delta S$ FOR LARGE $\boldsymbol{n}$}

Since $\delta E$ is exactly linear in $\delta \rho$, justification of our approximation requires only showing that $\delta S$ takes the form (2.7) for large $n$. This can be argued through a standard trick from statistical mechanics. ${ }^{9}$ One notes that the entropy

\footnotetext{
${ }^{9}$ We thank Mark Srednicki for explaining this argument to us.
}

$S=-\operatorname{Tr}(\rho \ln \rho)$ is -1 times the order $\epsilon$ term in the expansion of $\operatorname{Tr} \rho^{1+\epsilon}=\operatorname{Tr} \rho e^{\epsilon \ln \rho}$.

Let us now compute the entropy of $\rho_{R 1}$. We note that this density matrix is an average of $n$ terms, each identical to $\rho_{R 0}$ except that the density matrix describing a certain subsystem has been changed. The average is over the label telling us which subsystem has been changed. Thus, if $\rho_{R 0}$ takes the form $\rho_{R 0}=\otimes_{i=1}^{n} A$, then we may write

$$
\rho_{R 1}=\frac{1}{n} \sum_{i} A \otimes \cdots \otimes A \otimes B \otimes A \otimes \cdots \otimes A
$$

where again the index $i$ tells us where to place the factor $B$.

It is straightforward to see that for integer $k \ll n$ we have

$$
\operatorname{Tr} \rho_{R 1}=\left[\operatorname{Tr}\left(A^{k-1} B\right)\right]^{k}\left[\operatorname{Tr}\left(A^{k}\right)\right]^{n-k}+O(1 / n),
$$

since the number of terms having more than one factor of $B$ in the same subsystem are $O(1 / n)$. The standard trick is to substitute $k=1+\epsilon$ and take the coefficient of $\epsilon$ as $(-1$ times) the entropy. This yields

$$
\operatorname{Tr} \rho_{R 1}=[\operatorname{Tr}[(1+\epsilon \ln A) B]]^{1+\epsilon}[\operatorname{Tr}(A(1+\epsilon \ln A))]^{n-1-\epsilon} .
$$

But $\operatorname{Tr} A=\operatorname{Tr} B=1$. So we have

$$
\begin{aligned}
\operatorname{Tr} \rho_{R 1} & =[1+\epsilon \operatorname{Tr}(B \ln A)][1+\epsilon(n-1) \operatorname{Tr}(A \ln A)] \\
& \approx 1+\epsilon[\operatorname{Tr}[(B-A) \ln A]+n \operatorname{Tr}(A \ln A)] .
\end{aligned}
$$

Recognizing $n \operatorname{Tr}(A \ln A)$ as -1 times the entropy of $\rho_{0}$, it follows that

$$
\delta S=-\operatorname{Tr}[(B-A) \ln A]=-\operatorname{Tr} \delta \rho \ln \rho_{R 0},
$$

where in the last step we have again used $\operatorname{Tr}(A-B)=0$.
[1] J.D. Bekenstein, Phys. Rev. D 7, 2333 (1973).

[2] S.W. Hawking, Commun. Math. Phys. 43, 199 (1975).

[3] For reviews consult, for example, J.M. Maldacena, "Black holes in string theory," hep-th/9607235; A.W. Peet, Class. Quantum Grav. 15, 3291 (1998).

[4] T. Jacobson, Phys. Rev. Lett. 75, 1260 (1995).

[5] T. Jacobson and R. Parentani, Found. Phys. 33, 323 (2003).

[6] T. Padmanabhan, Mod. Phys. Lett. A 17, 1147 (2002).

[7] J.D. Bekenstein, Phys. Rev. D 7, 2333 (1973); 9, 3292 (1974).

[8] J.D. Bekenstein, "Quantum information and quantum black holes," gr-qc/0107049.

[9] L. Susskind, J. Math. Phys. 36, 6377 (1995).

[10] G. 't Hooft, "Dimensional Reduction In Quantum Gravity," gr-qc/9310026.
[11] R.D. Sorkin, in Proceedings of the GR10 Conference, Padova, 1983, edited by B. Bertotti, F. de Felice, and A. Pascolini (Consiglio Nazionale della Ricerche, Roma, 1983), Vol. 2; L. Bombelli, R.K. Koul, J.H. Lee, and R.D. Sorkin, Phys. Rev. D 34, 373 (1986); M. Srednicki, Phys. Rev. Lett. 71, 666 (1993).

[12] R.M. Wald, Class. Quantum Grav. 16, A177 (1999).

[13] R.M. Wald, Living Rev. Relativ. 4, 6 (2001).

[14] D. Marolf and R. Sorkin, Phys. Rev. D 66, 104004 (2002).

[15] D. Marolf and R. Sorkin, Phys. Rev. D 69, 024014 (2004).

[16] R.D. Sorkin, "The statistical mechanics of black hole thermodynamics," gr-qc/9705006.

[17] W.G. Unruh, Phys. Rev. D 14, 870 (1976).

[18] D.W. Sciama, P. Candelas, and D. Deutsch, Adv. Phys. 30, 327 (1981). 